\title{
A single-step genome wide association study on Body Size Traits using imputation-based whole- genome sequence data in Yorkshire pigs
}

Huatao Liu

China Agricultural University College of Animal Science and Technology

Hailiang Song

China Agricultural University College of Animal Science and Technology

Yifan Jiang

China Agricultural University College of Animal Science and Technology

Yao Jiang

China Agricultural University College of Animal Science and Technology

Fengxia Zhang

China Agricultural University College of Animal Science and Technology

Yibing Liu

China Agricultural University College of Animal Science and Technology

Yong Shi

China Agricultural University College of Animal Science and Technology

Xiangdong Ding

China Agricultural University

Chuduan Wang ( $\sim$ cdwang@cau.edu.cn )

China Agricultural University

\section{Research}

Keywords: pigs, body size traits, ssGWAS

Posted Date: November 9th, 2020

DOl: https://doi.org/10.21203/rs.3.rs-101177/v1

License: (c) (i) This work is licensed under a Creative Commons Attribution 4.0 International License.

Read Full License 


\section{A single-step genome wide association study on Body}

sequence data in Yorkshire pigs

4 Huatao Liu, Hailiang Song, Yifan Jiang, Yao Jiang, Fengxia Zhang, Yibing Liu, Yong

5 Shi, Xiangdong Ding ${ }^{*}$, Chuduan Wang*

6 National Engineering Laboratory for Animal Breeding, Laboratory of Animal

7 Genetics, Breeding and Reproduction, Ministry of Agriculture, College of Animal

8 Science and Technology, China Agricultural University, Beijing 100193, P.R. China;

10 Email addresses:

11 Huatao Liu, 925457179@qq.com

12 Hailiang Song, 530638625@qq.ocm

13 Yifan Jiang, jiangyf2020_2020@163.com

14 Yao Jiang, jiangyao133996@126.com

15 Fengxia Zhang, 895315294@qq.com

16 Yibing Liu, 279547013@qq.com

17 Yong Shi, 2273190789@qq.com

18 Xiangdong Ding, xding@cau.edu.cn

19 Chuduan Wang, cdwang@ cau.edu.cn 


\section{Abstract}

Background: The body shape of pig is the most direct production index of pig, which can fully reflect the growth status of pig and is closely related to some important economic traits. In this study, genome-wide association study on seven body size traits, the body length (BL), height $(\mathrm{BH})$, chest circumference $(\mathrm{CC})$, abdominal circumference $(\mathrm{AC})$, cannon bone circumference (CBC), rump width (RW) and chest width $(\mathrm{CW})$ were conducted in Yorkshire pigs.

Methods: Illumina Porcine $80 \mathrm{~K}$ SNP chip were used to genotype 589 of 5,572 Yorkshire pigs with body size records, and then the chip data was imputed to sequencing data. After quality control of imputed sequencing data, 784,267 SNPs were obtained, and the averaged linkage disequilibrium $\left(\mathrm{r}^{2}\right)$ was 0.191 . We used the single-trait model and the two-trait model to conduct single-step genome wide association study (ssGWAS) on seven body size traits.

Results: A total of 198 significant SNPS were finally identified according to the P value and the contribution to the genetic variance of individual SNP. 11 candidate genes (CDH13, SIL2, CDC14A, TMRPSS15, TRAPPC9, CTNND2, KDM6B, CHD3, MUC13, MAPK4 and HMGA1) were found to be associated with body size traits in pigs, KDM6B and $\mathrm{CHD} 3$ jointly affect $\mathrm{AC}$ and $\mathrm{CC}$, and $\mathrm{MUC13}$ jointly affect RW and CW. These genes are involved in the regulation of bone growth and development as well as the absorption of nutrients and are associated with obesity. HMGA1 is proposed as strong candidate gene for body size traits because of its 
important function and high consistency with other studies regarding the regulation of body size traits. Our results could provide valuable information for pig breeding based on molecular breeding.

Keywords: pigs, body size traits, ssGWAS

\section{Introduction}

Pork is widely used as an important animal protein resource and has become one of the main sources of human protein. Commercial pigs (e.g. Duroc, Yorkshire and Landrace pigs) have the characteristics of fast growth, high feed utilization rate, high lean meat rate and obvious economic benefits. Therefore, it is not only a large number of breeding production, but also the focus of research. The body size trait is one kind of important phenotypic trait that can reflect the overall appearance of animals. Compared with the description of physical appearance, body size traits can objectively reflect the response of pigs to environment and other aspects[35]. In pig breeding, the body shape character index is often used as the most direct production index of pig. Body size is a typical quantitative (or complex) trait, understanding the genetic mechanism of body size differences among individuals can effectively help control the growth and production of animals[34]. At present, there are many researches on genetic parameters of pig external traits, which accelerate the process of genetic improvement of related traits. With the development of molecular 
61 biotechnology, many studies have been carried out to clarify the genetic basis of pig 62 body size traits.

63 By far, 1172 QTLs have been found related to body size traits in pigs according to 64 PigQTLdb database (http://www.genome.iastate.edu/cgibin/QTLdb/ss/index).

65 Although a range of researches have been done in QTL mapping, wide confidence 66 intervals (covering more than $20 \mathrm{CM}$ ) for the positions of QTL remain that have 67 rarely been replicated[39; 42]. A new research era was initiated with advances in 68 single nucleotide polymorphism (SNP) chip and sequencing technology, and genome 69 wide association study (GWAS) has become one of the most efficient methods to 70 detect genetic variation in livestock[30]. Compared with traditional QTL localization, 71 GWAS has more advantages in mining the intensity of medium-potency variation 72 sites and defining the accuracy of genome segments containing variation sites[19; 26;

73 38; 41]. Although a large number of genome-wide association studies have been 74 carried out in pigs, only few GWAS focused on identifying genes related to external 75 traits. In particular, the investigation on body height, cannon bone circumference, 76 rump width and other important body size traits are still lacking.

77 Marker density is one key factor affecting the efficiency of GWAS as gene mapping 78 mainly relies on the linkage disequilibrium between causal mutation and markers[9].

79 Whole genome sequence data can definitely meet such requirements. In recent years, 80 with the rapid development of the new generation of sequencing technology, the cost 
81 of sequencing has been reduced rapidly, on one hand, a large number of samples and

82 the subsequent processing of sequence data are still time-consuming and costly,

83 limiting its utilization in genetic analysis. On the other hand, genotype imputation

84 provides one efficient tool to improve the marker density of SNP chip based on

85 sequence data. It can accurately predict the genotypes of polymorphic sites not

86 covered by the widely used SNP chip, allowing more genetic loci to be applied to

87 association analysis and improving the possibility of discovering new pathogenic

88 genes [32; 45].In this study, we used imputation-based whole genome sequence data

89 to carry out GWAS on seven body size traits in pigs.

90 Materials and methods

$91 \quad$ Ethics statement

92 The whole recording procedure of ear tissue samples was carried out in strict

93 accordance with the protocol approved by the Institutional Animal Care and Use

94 Committee (IACUC) at the China Agricultural University. The IACUC of the China

95 Agricultural University approved this study (permit number DK996).

\section{Animals and phenotypes}

97 Yorkshire pigs born 2013-2016 from one pig breeding farm in Beijing were collected

98 in this study. Performance test on seven body size traits were carried out at the body

99 weight of about $100 \mathrm{~kg}$ for pigs. In total, 5,572 Yorkshire pigs with phenotypic 
records and pedigree information were selected. The seven body size traits included

101 body length (BL), body height $(\mathrm{BH})$, chest circumference (CC), abdominal

102 circumference $(\mathrm{AC})$, cannon bone circumference $(\mathrm{CBC})$, chest width $(\mathrm{CW})$ and rump

103 width (RW). Table 1 presents the descriptive statistics of body weight and seven body

104 size traits. There were 4898 records for AC and 5572 records for the other six body

105 size traits and body weight. Normal test showed all the traits followed normal

106 distribution, and the body weight had the largest standard deviation of 12.59 and

107 coefficient of variation of $(12.43 \%)$, it was used as a covariate considering its

108 influence on the body size traits in further analysis.

109 Genotype data and imputation

110 In this study, 589 out of 5572 Yorkshire pigs with body size records were genotyped

111 using the PorcineSNP80 Bead Chip (Illumina, San Diego, CA), which includes

11268,528 SNPs across the whole pig genome. In order to improve the marker density,

113 the genotyped animals with another 6103 pigs genotyped with PorcineSNP80 [43]

114 were imputed to whole genome sequence data using Beagle 4.1[10]. A wide

115 collection of 289 sequenced pigs all with average sequencing depth of $\sim 25 \mathrm{X}$ from 6

116 different pig breeds were used as reference data for imputation and each breed

117 contained 24 to 94 pigs. The composition of reference data and the SNP calling of

118 these individuals were described by Yan et al.[54]. After SNP calling, 46,766,110

119 SNPs were retained as the reference panel for imputation. On average, the genotype

120 concordance rate across all variants was $92 \%$, which is sufficient for further 
121 analysis[43]. After imputation, in this study, the following genotype quality control

122 procedure was carried out using the PLINK software (v1.90)[36]. (1) SNPs with

123 minor allele frequency (MAF) lower than 0.01 and deviated from Hardy - Weinberg

124 equilibrium $\left(\mathrm{P}<10^{-6}\right)$ were excluded and only variants located on autosomes were

125 used for further analysis;(2) the SNP with call rate less than 0.95 were removed;(3)

126 individuals with call rate less than 0.90 were excluded. In addition, in order to

127 decrease the influence of the dependence of adjacent markers on the high false

128 positive of GWAS analysis, the SNP were further pruned, the SNP with linkage

129 disequilibrium $\left(\mathrm{r}^{2}\right)$ in slide window of 50 SNPs less than 0.9 were selected. Finally, all

130 the genotyped animals and 784267 SNPs were retained.

\section{Statistical models}

\section{2 genetic correlation}

133 According to the information of 5,572 pigs in this study, the restricted maximum

134 likelihood method (AI-REML) in DMU v6.0 software[31] was used to estimate the

135 genetic correlations of seven body size traits.

136 The animal model was used to estimate the genetic parameters:

$$
\mathbf{y}=\mu+X b+Z_{1} \mathbf{a}+\mathbf{Z}_{2} \mathbf{t}+\mathbf{e}
$$

138 with 


$$
\mathbf{E}\left\{\begin{array}{l}
\mathbf{y} \\
\mathbf{a} \\
\mathbf{t} \\
\mathbf{e}
\end{array}\right\}=\left\{\begin{array}{l}
\mathbf{X b} \\
\mathbf{0} \\
\mathbf{0} \\
\mathbf{0}
\end{array}\right\}, \operatorname{Var}\left\{\begin{array}{l}
\mathbf{a} \\
\mathbf{t} \\
\mathbf{e}
\end{array}\right\}=\left\{\begin{array}{rrr}
A \sigma_{\mathbf{a}}^{2} & \mathbf{0} & \mathbf{0} \\
& \mathbf{I} \sigma_{\mathbf{t}}^{2} & \mathbf{0} \\
& & \mathbf{I} \sigma_{\mathrm{e}}^{2}
\end{array}\right\}
$$

140 where, $\mathbf{y}$ is the vector of phenotypic values of each body size trait; $\boldsymbol{\mu}$ is the population

141 mean; $\mathbf{b}$ is the fixed effect of herd-year-season; $\mathbf{a}$ is the vector of additive genetic

142 effects; $\mathbf{t}$ is the covariate vector of body weight effects; $\mathbf{e}$ is a vector of residual

143 effects. $\mathbf{X}, \mathbf{Z}_{\mathbf{1}}$ and $\mathbf{Z}_{\mathbf{2}}$ are incidence matrices associating $\mathbf{b}, \mathbf{a}$ and $\mathbf{t}$ with $\mathbf{y}$,

144 respectively. A is the genetic relationship matrix, five generations of pedigree were

145 traced back to construct $\mathrm{A}$, and $\boldsymbol{\sigma}_{\mathbf{a}}{ }^{2}$ is the additive genetic variance. $\mathbf{I}$ is the identity

146 matrix of appropriate dimension, $\boldsymbol{\sigma}_{\mathrm{t}}{ }^{2}$ is the variance of body weight effect and $\boldsymbol{\sigma}_{\mathrm{e}}{ }^{2}$ is

147 the residual variance.

148 Subsequently, genetic correlations were calculated based on the variance components

149 as follows:

$$
\mathbf{r}_{\mathrm{A}}=\frac{\operatorname{cov}\left(\mathrm{a}_{1}, \mathbf{a}_{2}\right)}{\sigma_{\mathrm{a}_{1}} \sigma_{\mathrm{a}_{2}}}
$$

151 where, $\mathrm{r}_{\mathrm{A}}$ is the genetic correlation between trait 1 and trait $2, \mathrm{a}_{1}$ and $\mathrm{a}_{2}$ represent the

152 additive genetic values of trait 1 and trait 2 for same individuals, cov (a1, a2) and бa1,

153 бa2 refer to the genetic covariance of two traits and the genetic standard deviation of

154 trait 1 and trait 2 , respectively. 
156 In this study, single-step GWAS (ssGWAS), which can simultaneously use all the

157 SNP information and utilize the ungenotyped animals with phenotypic records[47],

158 was implemented to identify significant SNPs associated with body size traits.

159 Considering the genetic correlations between body size traits, two-trait ssGWAS

160 model was also conducted on traits with high genetic correlations.

161 Single-trait ssGWAS

162 Single-trait ssGWAS model was used for three body size traits BL,BH and CBC.

$$
\mathbf{y}=\mathbf{X b}+\gamma \mathbf{W}+\mathbf{Z g}+\mathbf{e}
$$

163 where $\mathbf{y}$ is the vector of phenotypic values, $\mathbf{b}$ is the vector of fixed effects including

164 herd-year-season-sex, $\mathbf{W}$ is the covariate of body weight, $\mathbf{g}$ is the vector of additive

165 genetic effects, following a normal distribution of $\mathrm{N}\left(\mathbf{0}, \mathbf{H}{\sigma_{g}}^{2}\right)$, in which $\mathbf{H}$ is the

166 matrix of additive genetic relationships incorporating both pedigree and genomic

167 information, $\sigma_{g}{ }^{2}$ is the additive genetic variance, $\mathbf{e}$ is the vector of random

168 residuals with distribution of $\mathrm{N}\left(0, \mathbf{I} \sigma_{e}{ }^{2}\right)$, in which $\mathbf{I}$ is the identity and $\sigma_{e}{ }^{2}$ is the

169 residual variance. $\mathbf{X}, \mathbf{W}$ and $\mathbf{Z}$ is the incidence matrix associating $\mathbf{b}, \mathbf{w}, \mathbf{g}$ with $\mathbf{y}$,

170 respectively.

171 The genotyped and ungenotyped animals were considered simultaneously based on a

$172 \mathrm{H}$ matrix[4]. The inverse of the $\mathrm{H}$ matrix was written as follows: 


$$
H^{-1}=\left[\begin{array}{cc}
0 & 0 \\
0 & G_{w}^{-1}-A_{22}^{-1}
\end{array}\right]+A^{-1}
$$

173 where $\boldsymbol{A}^{\mathbf{- 1}}$ is the inverse of the numerator relationship matrix, $\boldsymbol{A}_{\mathbf{2 2}}^{\mathbf{- 1}}$ is only the

174 inverse of the pedigree-based relationship matrix for the genotyped animals, and $\boldsymbol{G}_{\boldsymbol{w}}^{-1}$

175 is the inverse of the genomic relationship matrix;, G weight markers were obtained by

176 reciprocals of expected marker variance[46].

177 The SNP effects could be estimated by ssGWAS. The proportion of genetic variance

178 explained by single SNP was calculated as follows:

$$
\frac{\operatorname{Var}\left(Z_{j} \hat{u}_{j}\right)}{\sigma_{a}^{2}} \times 100 \%
$$

179 where $\sigma_{a}{ }^{2}$ is the total genetic variance, $Z_{j}$ is a vector of the gene content of the jth

180 SNP for all animals, and $\hat{u}_{j}$ is the estimated marker effect of the jth SNP.

181 Two-trait ssGWAS

182 According to the genetic correlation estimations, four body size traits with high

183 genetic correlations ( $\mathrm{CC}$ and $\mathrm{AC}, \mathrm{RW}$ and $\mathrm{CW}$ ) were carried out using two-trait

184 ssGWAS model.

$$
\left[\begin{array}{l}
y_{1} \\
y_{2}
\end{array}\right]=\left[\begin{array}{cc}
X_{1} & 0 \\
0 & X_{2}
\end{array}\right]\left[\begin{array}{l}
b_{1} \\
b_{2}
\end{array}\right]+\left[\begin{array}{l}
\gamma_{1} \\
\gamma_{2}
\end{array}\right]\left[\begin{array}{l}
W_{1} \\
W_{2}
\end{array}\right]+\left[\begin{array}{cc}
Z_{1} & 0 \\
0 & Z_{2}
\end{array}\right]\left[\begin{array}{l}
g_{1} \\
g_{2}
\end{array}\right]+\left[\begin{array}{l}
e_{1} \\
e_{2}
\end{array}\right]
$$

186 where $\left[\begin{array}{l}y_{1} \\ y_{2}\end{array}\right]$ is the vector of observation values of trait I and II, $\boldsymbol{b}_{1}$ and $\boldsymbol{b}_{2}$ are the 187 vector of fixed effects of herd-year-season-sex of trait I and II, $\boldsymbol{X}_{\mathbf{1}}$ and $\boldsymbol{X}_{\mathbf{2}}$ are the 188 incidence matrix associating $\boldsymbol{b}_{1}$ and $\boldsymbol{b}_{2}$ with $\boldsymbol{y}_{\mathbf{1}}$ and $\boldsymbol{y}_{2},\left[\begin{array}{l}\boldsymbol{W}_{1} \\ \boldsymbol{W}_{2}\end{array}\right]$ is the vector of 
190 associating $\boldsymbol{W}_{\mathbf{1}}$ and $\boldsymbol{W}_{\mathbf{2}},\left[\begin{array}{l}\boldsymbol{g}_{\mathbf{1}} \\ \boldsymbol{g}_{\mathbf{2}}\end{array}\right]$ is the vector of additive genetic effects of the two 191 traits, following a normal distribution of $\mathrm{N}(\mathbf{0}, \mathbf{H} \otimes \mathbf{M})$, where $\mathbf{M}=\left[\begin{array}{cc}\sigma_{g 1}^{2} & \sigma_{g 12}^{2} \\ \sigma_{g 12}^{2} & \sigma_{g 2}^{2}\end{array}\right]$ is the 192 additive genetic variance and covariance matrix of the two traits, $\boldsymbol{Z}_{\mathbf{1}}$ and $\boldsymbol{Z}_{\mathbf{2}}$ are the 193 incidence matrix associating $\boldsymbol{g}_{\mathbf{1}}$ and $\boldsymbol{g}_{2}$ with $\boldsymbol{y}_{\mathbf{1}}$ and $\boldsymbol{y}_{2},\left[\begin{array}{l}\boldsymbol{e}_{1} \\ \boldsymbol{e}_{2}\end{array}\right]$ is the vector of 194 random errors with distribution of $\mathrm{N}(\mathbf{0}, \mathbf{I} \otimes \mathbf{R})$, where $\mathbf{I}$ is the identity matrix and $195 \mathrm{R}=\left[\begin{array}{cc}\sigma_{e 1}^{2} & \sigma_{e 12}^{2} \\ \sigma_{e 12}^{2} & \sigma_{e 2}^{2}\end{array}\right]$ is the residual variance and covariance matrix of the two traits.

196 In this study, for both single trait model and two-trait model of ssGWAS, blupf90

197 [5]was implemented to estimate genomic breeding values (GEBV), and afterwards, 198 based on GEBV, SNP effects and P-values were estimated via postGSf90.The P value 199 of each marker was calculated as follows[3]:

$$
\boldsymbol{P}_{\boldsymbol{i}}=\boldsymbol{P}_{t}\left(\frac{\widehat{u}_{i}}{\sqrt{\hat{\boldsymbol{\sigma}}_{i}^{2} / \boldsymbol{n}}}, \boldsymbol{n}-\mathbf{1}\right)
$$

201 where $\boldsymbol{P}_{\boldsymbol{i}}$ is the distribution function of $\mathrm{t}$ distribution, $\hat{u}_{i}$ is ith SNP effect, $\hat{\boldsymbol{\sigma}}_{\boldsymbol{i}}^{2}$ is the 202 genetic variance of ith SNP, $\mathrm{n}$ is the number of animals with ith SNP. In addition, the 203 proportion of genetic variance explained by the ith SNP could also be calculated as $204 \hat{\boldsymbol{\sigma}}_{\boldsymbol{i}}^{2} / \boldsymbol{\sigma}_{\boldsymbol{g}}^{2}$. Manhattan plots of SNP variance were obtained by the "qqman" $\mathrm{R}$ 205 package[14].

206 In order to control false positives, the False Discovery Rate (FDR)[6; 52] method for 207 multiple testing was used as follow: 
209 where $m$ is the number of times to be tested, $n$ is the number of significant SNPs at

210 assigned FDR level, e.g. 0.05. $\mathrm{P}_{\mathrm{Max}}$ is the genome-wide significance level empirical

211 P-value of FDR adjusted. Based on the P-values of SNPs obtained by ssGWAS, the

212 empirical P-value of FDR adjusted at the genome-wide significance level of 0.05 was

213 calculated on each trait in this study.

\section{Identification of candidate genes}

215 After identifying significant SNPs by ssGWAS, the genes located in the $50 \mathrm{~Kb}$

216 downstream and $50 \mathrm{~Kb}$ upstream region of the significant SNPs were determined

217 using BedTools[37] and pig reference gene annotation

218 (http://www.ensembl.org/Sus_scrofa/Info/Index/; Sus scrofa 11.1 genome version).

219 Using the R package bioconductor (http://www.bioconductor.org/) to identify the 220 related pathways and functional annotation. QTLdb 221 (http://www.animalgenome.org/cgi-bin/QTLdb/SS/download?file= gbpSS_11.1) was 222 used to annotate significant SNPs located in previously mapped QTLs in pigs. R 223 package 'Cluster Profiler'[55] was used to carry out Gene Ontology (GO) and Kyoto 224 research on annotated candidate genes Encyclopedia of Genes and Genomes (KEGG) 225 enrichment analysis.

\section{Results}


228 Table 2 shows the genetic correlations of seven body size traits. The genetic 229 correlations ranged from -0.286 to 0.840 with standard errors ranging from 0.028 to 230 0.106. Among the seven body size traits, chest circumference (CC) and abdominal 231 circumference (AC), chest width (CW) and rump width (RW) had the higher genetic 232 correlations of 0.747 and 0.840 with standard errors of 0.055 and 0.028 , respectively.

233 The genetic correlations between other traits were lower than 0.3 , and some traits 234 were almost not genetic correlated with other traits, e.g. body length (BL) had very 235 low genetic correlation of $-0.010,0.03,-0.01,0.01$ with body height $(\mathrm{BH}), \mathrm{CC}, \mathrm{AC}$, 236 CW, respectively.

\section{Identification of significant SNPs associated with body size traits}

238 Two criteria of $\mathrm{P}$ value and SNP effect were respectively used to determine the SNPs

239 associated with body size traits. As to the $\mathrm{P}$ value, after the 0.05 significance level of 240 the whole genome was adjusted, the $\mathrm{P}_{\text {Max }}$ values of FDR-based multiple tests were $2419.26 \mathrm{E}-06$ for BL, 1.08E-05 for BH, 1.02E-05 for CBC, 9.74E-06 for AC, 1.05E-05 for 242 CC, 9.60E-06 for RW, and 1.01E-05 for CW. As shown in Table 3, a total of 88 243 significant SNPs was identified for seven body size traits. The Manhattan plots of the 244 three traits BL, BH and CBC using the single trait model are shown in Figure 1. For 245 BL, a total of 9 significant SNPs reached the genome-wide significance level, totally 246 accounting for $0.0085 \%$ of the genetic variance. These significant SNPS were located 
248 SSC17:33632497 explained the largest genetic variance $(0.0029 \%)$. For BH, only 6

249 SNPs were genome-wide significant, accounting for a total of $0.0123 \%$ of genetic

250 variance. They were located on SSC3, SSC5, SSC14, and SSC16.The interpretation of

251 scc16: 886074 has the largest genetic variance (0.0082\%). For $\mathrm{CBC}$, there were 15

252 significant SNPs at the genome-wide level, which explained $0.0267 \%$ of the genetic

253 variance, and the most significant SNPs were closely located on SSC1. For the two

254 pairs of genetic correlated traits using the two-trait model, the Manhattan plots of AC

255 and CC, RW and CW are shown in Figure 2. In total, 8, 17, 9, and 24 SNPs were

256 identified associated with $\mathrm{AC}, \mathrm{CC}, \mathrm{RW}$, and $\mathrm{CW}$, respectively, and these SNPs

257 explained $0.0109 \%, 0.0242 \%, 0.0099 \%$ and $0.0281 \%$ of genetic variances for the

258 corresponding traits. For each trait, the genetic variance explained by a single

259 significant SNP was very small, the largest of which for each trait were $0.0051 \%$

260 (SSC5:15137502), $0.0067 \%$ (SSC4:64552365), 0.0038\% (SSC9:2330339) and

$2610.0065 \%$ (SCC7:115471416), respectively. Although the genetic correlations existed

262 among seven body size traits, no common significant SNPs were found.

263 Considering the small contribution of above significant SNPs to the genetic variance,

264 the proportion of genetic variance explained by each SNP were also illustrated as

265 shown in Figure 3 in this study. Top 20 SNPs with the largest genetic variance were

266 selected for each trait(Table 3), SNPs for BL were located on SSC17, BH on SSC2,

267 SSC5 and SSC16, CBC on SSC7 and SSC4, SNPs with largest genetic variance for 
268 AC and CC are located on SSC12, those for RW and CW were on SCC6 SCC7,

269 SCC13 and SCC17. For each body size trait, BL, BH, CBC, AC, CC, RW and CW, 270 the top 20 SNPs explained $2.01 \%, 1.56 \%, 1.63 \%, 2.39 \%, 2.32 \%, 1.54 \%$ and $1.23 \%$ of

271 the genetic variance, respectively. Interestingly, the top 20 SNPs for AC and CC were 272 same, RW and CW shared half of the 20 SNPs. In total, 110 SNPs with larger 273 proportion of explanatory genetic variance were retained for further analysis (Stable 274 1).

\section{Identification of candidate genes}

276 All the significant SNPs identified by the two methods were annotated within the 50 $277 \mathrm{~Kb}$ downstream and upstream region with reference to the Sus scrofa 11.1 genome 278 assembly. According to the two methods of SNP significance and explained genetic 279 variance, 88 and 110 SNPs were identified without overlapping, and 64 and 40 genes 280 were found near these SNPs and only two of them were common, respectively (Table 2813 and Stable 1). Six and seven genes were found to be related to the corresponding 282 body size traits by the two methods. The biological processes and pathways involved 283 in these genes include calcium channel proteins, lipid metabolism, and cell 284 proliferation.

\section{Discussion}


287 Genotype marker density is one important factor affecting the efficiency of GWAS 288 [9].With the increase of marker density, the linkage disequilibrium between markers 289 and the target trait QTL is increased, it is helpful for QTL detection. In previous 290 studies, the advantages of whole genome sequencing data have been 291 demonstrated[49]. However, its high cost hampered the widely application of 292 sequencing data. Genotype imputation was proved efficiently to impute the SNP chip 293 data to sequencing data with high accuracy[20]. Our results indicated that 294 imputation-based WGS data dramatically improved the power of GWAS, among the 295 significant SNPs identified in this study, only 3 out of the 88 significant SNPs were 296 located in the PorcineSNP80 SNP chip, the remaining 85 loci were identified in the 297 sequencing data. Moreover, among the 110 non-repeating loci screened by 298 interpretation variance, 101 are new loci after imputation, which indicates that 299 imputed WGS data adds a lot of useful information

300 Increasing marker density could lead to high linkage disequilibrium (LD) to improve 301 the resolution of gene mapping, while it may also be a burden[24]. Too high LD 302 between markers will cause noise and increase false positive[50]. One of the 303 strategies to deal with such dilemma is to pre-select SNP, which can be done via SNP 304 selection to only keep a set of SNPs that are mutually uncorrelated[11; 18]. 305 Therefore, we pruned SNPs according to the genome-wide sequence data to reduce 306 the LD degree between SNPs, and retained the loci in the original 80K chip. In this 307 study, 44003 out of the qualified 50179 SNPs in PorcineSNP80 chip according to the 
genotype quality control were retained, and the average linkage disequilibrium of the

309 finally used 784,267 SNPs is similar to that of the chip data, the average $r^{2}$ was 0.191

310 and 0.195 , respectively. This not only retains the original SNPs but also increases a

311 large number of SNPS, and does not cause the increase of LD.

\section{The advantage of ssGWAS}

313 Single SNP regression model is widely used in GWAS to identify the association of

314 SNP with traits of interest, whereas it usually yields a high false-positive rate due to

315 ignoring the linkage disequilibrium between adjacent SNPs. Wang et al.[47] proposed

316 Single-step GWAS (ssGWAS) that combines all the data (genotype, phenotype and

317 pedigree information) in one step. It can simultaneously utilize all the markers

318 compared with the single-marker regression genome-wide association analysis,

319 resulting in higher power and accuracy[48]. In addition, ssGWAS is able to use

320 sliding windows to simultaneously analyze multiple SNPs to reduce errors[8; 17],

321 Wang et al. (2012) reported that ssGWAS achieved accuracy of $0.81 \pm 0.02$ using

3221500 genotype animals, which was more accurate than single SNP regression

323 model[47]. Moreover, ssGWAS can utilize more individuals, the sample size in this

324 study is not very large, but has a large number of phenotypic data of ungenotyped

325 animals. Compared with traditional GWAS, ssGWAS can make full use of this part of

326 information, expand the sample size to a certain extent, improve the accuracy of SNP

327 effect estimation, and further improve the efficiency of SNP identification. 
329 Theoretically, the SNPs with smallest $\mathrm{p}$ values were supposed to explain relatively

330 high proportion of genetic variance. Likewise, the SNPs with large effects should be

331 significantly associated with the trait of interest. However, our results indicated that

332 the SNPs with smallest P values did not have large effects, there was no overlap

333 between the top 20 SNPs with smallest P values and with largest SNP effects for each

334 trait. Therefore, in order to locate QTLs related to traits more accurately and

335 comprehensively, this study identified significant SNPs from both P value and SNP

336 effect. The proportion of genetic variance explained by most the significant SNPs was

337 small $(0.00004 \%-0.00653 \%)$ for all traits, and the maximum genetic variance of all

338 SNPs was also not large $(0.0557 \%-0.1205 \%)$, perhaps because too many SNPs were

339 used in the sequencing data in this study, leading to small effect of each related SNP

340 for each trait. It also indicates that SNPs controlling body size traits are widely

341 distributed on the genome, fitting well the infinitesimal model. It was reported that for

342 complex traits such as height, action sites are widely distributed across the entire

343 genome, indicating that almost all genes are involved in the regulation of height[7].

344 Pleiotropic effects can lead to genetic correlation between traits. From the aspect of P

345 value, no overlap of significant SNPs associated with two genetic related trait pairs

346 AC and CC, RW and CW were detected in this study. However, more common

347 SNPs with largest effects (not statistically significant) were found in each pair of 

completely overlapped, these SNPs were adjacent to each other and located near SCC12:53132997. Therefore, it is speculated that these SNPs constitute an important

351 QTL and jointly affect AC and CC. Similarly, there may be QTLs associated with 352 RW and CW around SSC6:39553559 and SCC13:135373704. In addition, we took 20

353 SNPs as a sliding window, and found that the top 20 windows with largest genetic 354 effects respective for $\mathrm{AC}$ and $\mathrm{CC}$ were overlapped, as well for RW and CW. The 355 above results further reflect 'one factor produces multiple effects', suggesting that 356 highly genetic related traits are probably regulated by the same QTL.

\section{Potential Candidate Genes for BL、BH and CBC}

358 The body length (BL) is an important index to investigate the breeding performance 359 of animals. According to bioinformatics analysis, CDH13 near SCC6:5671575 could 360 be used as a candidate gene affecting body length. CDH13 is a unique cadherin[44] 361 that regulates cell adhesion, signal transduction and cell growth[29], and plays an 362 important role in the formation of tissues and organs[22]. The ingestion and transfer 363 of Ca will affect the bone development of body for a long time[27], therefore, CDH13 364 has a certain influence on the growth and development of the body. For BH, SIL2 was 365 found to be associated with this trait near SCC2:46827557, Proteomic studies showed 366 that SIL1 elevation alters the expression of proteins including crucial players in 367 neurodegeneration, abnormal expression of SIL1 has an impact on the morphology of 
368 the body, which can reduce the body size[28]. CBC reflects the physical quality of the

369 animal, whether it is strong or not. There are three candidate genes associated with

370 CBC, CDC14A, TMPRSS15 and TRAPPC9. CDC14A is widely expressed in

371 eukaryotic cell biology of a special kind of highly conservative dual specificity

372 phosphatase, a variety of studies from yeast to human somatic cells have shown that

373 CDC14 involves extensive roles, including embryonic development and body size[1].

374 TMPRSS15 has an impact on the digestive efficiency of animals, and has been found

375 to be associated with the formation of cholesterol in humans and has been shown to

376 be associated with the development of fat and body weight in mice[51]. TMPRSS15

377 has also a higher variance ranking based on the SNP effect. The gene mutation of 378 transporter particle complex 9 (TRAPPC9), a protein subunit of transporter particle II

379 (TRAPPII), can lead to abnormal embryonic development, abnormal dietary behavior,

380 and is associated with body mass index[2;33].

\section{Potential Candidate Genes for AC and CC}

382 AC and CC are a pair of highly genetically-related body size traits, which determine

383 the body size of animals and are indicators to fatness and thinness. CTNND2 was

384 closely related to AC according to the P value. Studies have shown that CTNND2

385 participates in the regulation of cell proliferation and affects the body node number of

386 zebrafish[57]. It is found that KDM6B and CHD3 jointly affect AC and CC. KDM

387 subfamily 6 enzymes B (KAM6B) plays an important role in repression of 
developmental genes[25], and has a regulatory effect on chondrocyte differentiation,

389 thus affecting bone growth and development[15]. CDH3 is a calcium-binding protein

390 that is involved in calcium ion binding and protein binding and is associated with

391 diseases such as malnutrition and developmental malformations. Studies have shown

392 that CHD3 regulates the developmental morphology of zebrafish heart, thereby

393 affecting the abdominal circumference and body shape of zebrafish[12].

\section{Potential Candidate Genes for RW and CW}

395 For RW and CW, MUC13 was detected to affect both RW and CW. MUC13

396 promotes cell proliferation and migration, inhibits apoptosis, and reduces adhesion

397 through a number of signaling pathways[40], and has a certain effect on the 398 absorption of intestinal nutrients, thus affecting the growth and development of bone 399 and the organism. It was found that MAPK4 and HMGA1 affect RW and CW of pigs 400 respectively. MAPK4 is mitogen-activated protein kinase 4, which is involved in the 401 absorption and decomposition of sugars and the formation of fat, so it is related to 402 obesity traits[53]. HMGA1 affects the expression of two IGFBP(insulin-like growth 403 factor binding protein) protein species and plays an important role in cell growth and 404 differentiation[13; 21]. Studies have shown that the deletion of HMGA1 gene results 405 in a significant decrease in the body size of mice[16]. Moreover, a large number of 406 studies have shown that HMGA1 is related to the body size character of pigs. Ji et 407 al.[23] found HMGA1 was a candidate gene affecting body size of pig through 
409 pig limb cells and affects the growth and differentiation of chondrocytes. Because of 410 the functional importance of HMGA1 and several studies have shown that it is highly 411 associated with body size traits, it is worth being verified in the future.

\section{Conclusion}

413 In this study, among seven body size traits in pigs, $\mathrm{CC}$ and $\mathrm{AC}, \mathrm{CW}$ and $\mathrm{RW}$ were 414 highly genetic correlated with correlation of 0.747 and 0.840 , respectively. We 415 implemented ssGWAS to identify SNPs associated with body size traits based on two 416 aspects of $\mathrm{P}$ value and the proportion of explanatory genetic variance of SNP. In total, 417198 SNPs were identified associated with seven body size traits in Yorkshire, 418 correspondingly, 11 genes were related to body size traits, among which HMGA1 419 could be worth being validated in further study.

\section{Data availability}

421 The ped and the map are not publicly available because the genotyped animals belong 422 to commercial breeding companies, but are available from the corresponding author 423 on reasonable request.

\section{Conflict of interest}

425 The authors declare that they have no competing interest. 


\section{Author contributions}

427 XD and CD conceived and supervised the study. HT, HL and LF helped complete the 428 imputation of the chip data and provide technical guidance. HT, JY, HL, YF collected 429 the samples and recorded the phenotypes. JY and LF extracted the DNA for 430 genotyping. HT, FX, YB and SY contributed to the visualization of data. HT and XD 431 wrote and revised the manuscript. All authors read and approved the manuscript.

\section{$432 \quad$ Funding}

433 The authors are grateful to all the funding agencies. This work was supported by

434 grants for the National Key Research and Development Project (2019YFE0106800, 435 2018YFD0501000), China Agriculture Research System (CARS-35), Modern 436 Agriculture Science and Technology Key Project of Hebei Province (19226376D), the 437 National Natural Science Foundation of China (31671327).

\section{References:}

439 1. Research progress in function of cell division cycle protein Cdc14A. Journal of Jilin $440 \quad$ Univeristy. Medicine edition. 2018;44(1671-587X(2018)44:5<1105:XBFLZQ>2.0.TX;2-55): $441 \quad 1105-8$.

442 2. Abbasi AA, Blaesius K, Hu H, Latif Z, Picker-Minh S, Khan MN et al. Identification of a 443 novel homozygous TRAPPC9 gene mutation causing non-syndromic intellectual disability, 444 speech disorder, and secondary microcephaly. Am J Med Genet B Neuropsychiatr Genet. 
446 3. Aguilar I, Legarra A, Cardoso F, Masuda Y, Lourenco D, Misztal I. Frequentist p-values 447 for large-scale-single step genome-wide association, with an application to birth weight in 448 American Angus cattle. GENETICS SELECTION EVOLUTION. 2019;51(28). 449 doi:10.1186/s12711-019-0469-3.

450 4. Aguilar I, Misztal I, Johnson DL, Legarra A, Tsuruta S, Lawlor TJ. Hot topic: A unified 451 approach to utilize phenotypic, full pedigree, and genomic information for genetic evaluation 452 of Holstein final score. J Dairy Sci. 2010;93(2): 743-52. doi:10.3168/jds.2009-2730.

453 5. Aguilar I, Misztal I, Legarra A, Tsuruta S. Efficient computation of the genomic 454 relationship matrix and other matrices used in single-step evaluation. JOURNAL OF 455 ANIMAL BREEDING AND GENETICS. 2011;128(6SI): 422-8.

6. BENJAMINI Y, HOCHBERG Y. Controlling the false discovery rate - a practical and 460 powerful approach to multiple testing. JOURNAL OF THE ROYAL STATISTICAL SOCIETY SERIES B-STATISTICAL METHODOLOGY. 1995;57(1): 289-300.

461 7. Boyle EA, Li YI, Pritchard JK. An expanded view of complex traits: From polygenic to omnigenic. Cell. 2017;169(7): 1177-86. doi:10.1016/j.cell.2017.05.038.

463 8. Braz CU, Taylor JF, Bresolin T, Espigolan R, Feitosa F, Carvalheiro R et al. Sliding 464 window haplotype approaches overcome single SNP analysis limitations in identifying genes 465 for meat tenderness in Nelore cattle. BMC Genet. 2019;20(1): 8. 466 doi:10.1186/s12863-019-0713-4. 

and across Nordic cattle breeds. J Dairy Sci. 2012;95(11): 6795-800. doi:10.3168/jds.2012-5585.

10. Browning BL, Browning SR. A unified approach to genotype imputation and Haplotype-Phase inference for large data sets of trios and unrelated individuals. The American Journal of Human Genetics. 2009;84(2): 210-23. doi:10.1016/j.ajhg.2009.01.005.

11. Calus M, Vandenplas J. SNPrune: An efficient algorithm to prune large SNP array and sequence datasets based on high linkage disequilibrium. Genet Sel Evol. 2018;50(1): 34 . doi:10.1186/s12711-018-0404-z.

12. Cho SH, Lee CH, Gi E, Yim Y, Koh HJ, Kang K et al. The rice rolled fine striped (RFS) CHD3/Mi-2 chromatin remodeling factor epigenetically regulates genes involved in oxidative stress responses during leaf development. Front Plant Sci. 2018;9(364. doi:10.3389/fpls.2018.00364.

13. Cleynen I, Van de Ven WJ. The HMGA proteins: A myriad of functions (Review). Int J Oncol. 2008;32(2): 289-305.

14. D. Turner S. Qqman: An R package for visualizing GWAS results using Q-Q and manhattan plots. Journal of open source software. 2018;3(25): 731. doi:10.21105/joss.00731.

15. Dai J, Yu D, Wang $\mathrm{Y}$, Chen $\mathrm{Y}$, Sun $\mathrm{H}$, Zhang $\mathrm{X}$ et al. Kdm6b regulates cartilage development and homeostasis through anabolic metabolism. Ann Rheum Dis. 2017;76(7): 1295-303. doi:10.1136/annrheumdis-2016-210407. 

372-8. doi:10.1242/bio.20146759.

17. Guerra FP, Suren H, Holliday J, Richards JH, Fiehn O, Famula R et al. Exome resequencing and GWAS for growth, ecophysiology, and chemical and metabolomic composition of wood of Populus trichocarpa. BMC Genomics. 2019;20(1): 875. doi:10.1186/s12864-019-6160-9.

18. Hazelett DJ, Conti DV, Han Y, Al OA, Easton D, Eeles RA et al. Reducing GWAS complexity. Cell Cycle. 2016;15(1): 22-4. doi:10.1080/15384101.2015.1120928.

19. Hirschhorn JN, Daly MJ. Genome-wide association studies for common diseases and complex traits. Nature Reviews Genetics. 2005;6(2): 95-108. doi:10.1038/nrg1521.

20. Hoze C, Fouilloux MN, Venot E, Guillaume F, Dassonneville R, Fritz S et al. High-density marker imputation accuracy in sixteen French cattle breeds. Genet Sel Evol. 2013;45(33. doi:10.1186/1297-9686-45-33.

21. Hristov AC, Cope L, Di Cello F, Reyes MD, Singh M, Hillion JA et al. HMGA1 correlates with advanced tumor grade and decreased survival in pancreatic ductal adenocarcinoma. Modern Pathology. 2010;23(1 ): 98-104. doi:10.1038/modpathol.2009.139. TLR4 as a negative regulator of keratinocyte proliferation. PLoS One. 2017;12(10): e185668. doi:10.1371/journal.pone.0185668. 

patterns in large scale DNA based gene-gene interaction studies. BioData Min. 2019;12(11. doi:10.1186/s13040-019-0199-7.

25. Jones SE, Olsen L, Gajhede M. Structural Basis of Histone Demethylase KDM6B Histone doi:10.1021/acs.biochem.7b01152.

26. Klein RJHJ. Complement factor $\mathrm{h}$ polymorphism in Age-Related macular degeneration. Science. 2005;308): 385-9.

27. Kovacs CS. Maternal mineral and bone metabolism during pregnancy, lactation, and doi:10.1152/physrev.00027.2015. SIL1 increase: Taking a closer look beyond the consequences of elevated expression level. Mol Neurobiol. 2018;55(3): 2524-46. doi:10.1007/s12035-017-0494-6. suppressor genes and inhibition of expression of DNA methyltransferase 1 in non-small cell lung cancer. Exp Biol Med (Maywood). 2016;241(14): 1531-9. doi:10.1177/1535370216645211. 
prospects. Nature Reviews Genetics. 2009;10(8): 565-77. doi:10.1038/nrg2612.

531 31. Madsen PAJJ. A user's guide to DMU: A package for analysing multivariate mixed models. 2013.

32. Marchini J, Howie B. Genotype imputation for genome-wide association studies. NATURE REVIEWS GENETICS. 2010;11(7): 499-511. doi:10.1038/nrg2796.

33. Mbimba T, Hussein NJ, Najeed A, Safadi FF. TRAPPC9: Novel insights into its trafficking and signaling pathways in health and disease (Review). Int J Mol Med. 2018;42(6): 2991-7. doi:10.3892/ijmm.2018.3889.

34. Niu P, Kim S, Choi B, Kim T, Kim J, Kim K. Porcine insulin-like growth factor 1 (IGF1) gene polymorphisms are associated with body size variation. GENES \& GENOMICS.

35. Ohnishi C, Satoh M. Estimation of genetic parameters for performance and body measurement traits in Duroc pigs selected for average daily gain, loin muscle area, and backfat thickness. Livestock Science. 2018;214(161-6. doi:10.1016/j.livsci.2018.05.022. tool set for Whole-Genome association and Population-Based linkage analyses. The American Journal of Human Genetics. 2007;81(3): 559-75. doi:10.1086/519795.

37. Quinlan AR, Hall IM. BEDTools: A flexible suite of utilities for comparing genomic features. Bioinformatics. 2010;26(6): 841-2. doi:10.1093/bioinformatics/btq033. 
39. Schreiweis MA, Hester PY, Moody DE. Identification of quantitative trait loci associated with bone traits and body weight in an F2 resource population of chickens. Genetics Selection Evolution. 2005;37(6): 677-98. doi:10.1051/gse:2005023.

40. Sheng YH, Triyana S, Wang R, Das I, Gerloff K, Florin TH et al. MUC1 and MUC13 differentially regulate epithelial inflammation in response to inflammatory and infectious stimuli. Mucosal Immunol. 2013;6(3): 557-68. doi:10.1038/mi.2012.98.

41. Simon-Sanchez J, Schulte C, Bras JM, Sharma M, Gibbs JR, Berg D et al. Genome-wide association study reveals genetic risk underlying Parkinson's disease. Nat Genet. 2009;41(12): 1308-12. doi:10.1038/ng.487.

42. Soller M, Weigend S, Romanov MN, Dekkers JCM, Lamont SJ. Strategies to assess structural variation in the chicken genome and its associations with biodiversity and biological performance. Poultry Science. 2006;85(12 ): 2061-78. doi:10.1093/ps/85.12.2061.

43. Song H, Ye S, Jiang Y, Zhang Z, Zhang Q, Ding X. Using imputation-based whole-genome sequencing data to improve the accuracy of genomic prediction for combined populations in pigs. Genet Sel Evol. 2019;51(1): 58. doi:10.1186/s12711-019-0500-8.

44. Takeuchi T, Liang SB, Matsuyoshi N, Zhou S, Miyachi Y, Sonobe H et al. Loss of T-cadherin (CDH13, H-cadherin) expression in cutaneous squamous cell carcinoma. Lab Invest. 2002;82(8): 1023-9. doi:10.1097/01.lab.0000025391.35798.f1.

45. van Leeuwen EM, Kanterakis A, Deelen P, Kattenberg MV, Slagboom PE, de Bakker PIW et al. Population-specific genotype imputations using minimac or IMPUTE2. NATURE 
46. VanRaden PM. Efficient methods to compute genomic predictions. J Dairy Sci. 2008;91(11): 4414-23. doi:10.3168/jds.2007-0980.

47. Wang H, Misztal I, Aguilar I, Legarra A, Muir WM. Genome-wide association mapping including phenotypes from relatives without genotypes. Genet Res (Camb). 2012;94(2): 73-83. doi:10.1017/S0016672312000274.

48. Wang H, Misztal I, Aguilar I, Legarra A, Fernando RL, Vitezica Z et al. Genome-wide association mapping including phenotypes from relatives without genotypes in a single-step (ssGWAS) for 6-week body weight in broiler chickens. Front Genet. 2014;5(134. doi:10.3389/fgene.2014.00134.

49. Wang L, Zhang Y, Zhang T, Zhang L, Yan H, Liu X et al. Genotyping by sequencing doi:10.1111/age.12547.

50. Wang X, Elston RC, Zhu X. The meaning of interaction. Hum Hered. 2010;70(4): 269-77. doi:10.1159/000321967.

51. Wang Z, Xu S, Du K, Huang F, Chen Z, Zhou K et al. Evolution of digestive enzymes and RNASE1 provides insights into dietary switch of cetaceans. Mol Biol Evol. 2016;33(12): multiple comparisons in the genetic dissection of complex traits. GENETICS. 1998;150(4): 
593 53. Wu YS, Chen YT, Bao YT, Li ZM, Zhou XJ, He JN et al. Identification and verification of 594 potential therapeutic target genes in Berberine-Treated zucker diabetic fatty rats through bioinformatics analysis. PLoS One. 2016;11(11): e166378. doi:10.1371/journal.pone.0166378. sequence association study rediscovered the missing QTL for lumbar number in sutai pigs. Sci Rep. 2017;7(1): 615. doi:10.1038/s41598-017-00729-0.

600

55. Yu G, Wang LG, Han Y, He QY. ClusterProfiler: An R package for comparing biological

601 themes among gene clusters. OMICS. 2012;16(5): 284-7. doi:10.1089/omi.2011.0118.

602 56. Zhang LC, Li N, Liu X, Liang J, Yan H, Zhao KB et al. A genome-wide association study 603 of limb bone length using a Large White x Minzhu intercross population. Genet Sel Evol. 2014;46(56. doi:10.1186/s12711-014-0056-6.

605 57. Zhang Y, Zhang R, Ding X, Ai K. EFNB2 acts as the target of miR-557 to facilitate cell 606 proliferation, migration and invasion in pancreatic ductal adenocarcinoma by bioinformatics 607 analysis and verification. Am J Transl Res. 2018;10(11): 3514-28. 
611 Figure 1. Manhattan plot of the genome-wide association study on three body

612 size traits by using single-trait model ssGWAS. BL, Body length; BH, Body height;

613 CBC, Cannon bone circumference. In the Manhattan plots, negative $\log 10 \mathrm{P}$-values of

614 the quantified SNPs were plotted against their genomic positions. The $\mathrm{x}$-axis

615 represents the chromosomes, and the y-axis represents the observed -log10(P-value).

616 Different colors indicate various chromosomes. Each trait has a significant threshold

617 of FDR adjusted, for (A) BL, it was $9.26 \times 10^{-6}$. Similarly, (B) BH was $1.08 \times 10^{-5}$,

618 and (D) $\mathrm{CBC}$ was $1.02 \times 10^{-5}$.

619 Figure 2. Manhattan plot of the genome-wide association study on four body size 620 traits by using two-trait model ssGWAS.

621 AC, Abdominal circumference; CC, Chest circumference; RW, Rump width; CW,

622 Chest width. AC and CC are a pair of traits, RW and CW are a pair of traits.

623 In the Manhattan plots, negative $\log 10$ P-values of the quantified SNPs were plotted

624 against their genomic positions. The $\mathrm{x}$-axis represents the chromosomes, and the

$625 \mathrm{y}$-axis represents the observed $-\log 10(\mathrm{P}$-value). Different colors indicate various

626 chromosomes. Each trait has a significant threshold of FDR adjusted, for (A) AC, it

627 was $9.74 \times 10^{-6}$. Similarly, (B) CC was $1.05 \times 10^{-5}$, (C) RW was $9.60 \times 10^{-6}$, and (D)

$628 \mathrm{CW}$ was $1.01 \times 10^{-5}$.

629 Figure 3. Manhattan plot of the genome-wide association study on seven body

630 size traits and Venn plot of SNPs according to the contribution of SNP to genetic

631 variance by using ssGWAS.

632 BL, Body length; BH, Body height; CBC, Cannon bone circumference; AC,

633 Abdominal circumference; $\mathrm{CC}$, Chest circumference; RW, Rump width; CW, Chest

634 width. BL, BH and $\mathrm{CBC}$ were single-trait models, $\mathrm{AC}, \mathrm{CC}, \mathrm{RW}$ and $\mathrm{CW}$ were

635 two-trait models. $\mathrm{AC}$ and $\mathrm{CC}$ are a pair of traits, $\mathrm{RW}$ and $\mathrm{CW}$ are a pair of traits.

636 In the Manhattan plots(A-G), the proportion of genetic variance of the quantified

637 SNPs were plotted against their genomic positions. The $\mathrm{x}$-axis represents the

638 chromosomes, and the y-axis represents the percentage of SNP explaining the genetic

639 variance. Different colors indicate different chromosomes.

640 Venn plot $(\mathrm{H})$ of SNPs for the two pairs of body size traits, AC and CC, RW and CW

641 are a pair of traits, respectively. 


\section{Tables}

643 Table 1 Descriptive statistics for body weight and seven body size traits

\begin{tabular}{ccccccc}
\hline Trait $^{1}$ & N-obs $^{2}$ & Mean & S.D. & CV $(\%)$ & Min value & Max value \\
\hline $\mathrm{BL}(\mathrm{cm})$ & 5573 & 108.89 & 6.18 & 5.67 & 88 & 134 \\
$\mathrm{BH}(\mathrm{cm})$ & 5573 & 62.87 & 2.92 & 4.64 & 51 & 75 \\
$\mathrm{CC}(\mathrm{cm})$ & 5573 & 104.58 & 5.75 & 5.50 & 85 & 126 \\
$\mathrm{AC}(\mathrm{cm})$ & 4898 & 113.52 & 6.31 & 5.56 & 94 & 137 \\
$\mathrm{CW}(\mathrm{cm})$ & 5572 & 29.75 & 2.31 & 7.76 & 19 & 38 \\
$\mathrm{RW}(\mathrm{cm})$ & 5573 & 31.64 & 2.13 & 6.73 & 22 & 40 \\
$\mathrm{CBC}(\mathrm{cm})$ & 5573 & 17.98 & 1.03 & 5.73 & 13 & 23 \\
$\mathrm{BW}(\mathrm{kg})$ & 5573 & 101.31 & 12.59 & 12.43 & 61 & 150 \\
\hline
\end{tabular}

644 Note: ${ }^{1} \mathrm{BL}=$ body length, $\mathrm{BH}=$ body height, $\mathrm{CC}=$ chest circumference, $\mathrm{AC}=$ abdominal

645 circumference, $\mathrm{CBC}=$ cannon bone circumference, $\mathrm{RW}=$ rump width, $\mathrm{CW}=$ chest

646 width,${ }^{2} \mathrm{~N}-\mathrm{obs}=$ number of observations

647 Table 2 Genetic correlations between seven body size traits

$\begin{array}{lllllllll}\text { Trait }^{1} & \text { BL } & \text { BH } & \text { CC } & \text { AC } & \text { CW } & \text { RW } & \text { CBC }\end{array}$

\begin{tabular}{|c|c|c|c|c|c|c|}
\hline $\mathrm{BL}$ & $-0.010(0.088)$ & $0.033(0.092)$ & $-0.014(0.092)$ & $0.014(0.078)$ & $-0.286(0.078)$ & $0.206(0.078)$ \\
\hline $\mathrm{BH}$ & & $0.171(0.104)$ & $0.071(0.106)$ & $-0.221(0.091)$ & $-0.217(0.090)$ & $-0.105(0.096)$ \\
\hline $\mathrm{CC}$ & & & $0.747(0.055)$ & $0.255(0.093)$ & $0.127(0.095)$ & $0.197(0.096)$ \\
\hline $\mathrm{CW}$ & & & & & $0.840(0.028)$ & $0.015(0.086)$ \\
\hline RW & & & & & & $-0.032(0.085)$ \\
\hline
\end{tabular}

$\mathrm{CBC}$

648 Note: ${ }^{1} \mathrm{BL}=$ body length, $\mathrm{BH}=$ body height, $\mathrm{CC}=$ chest circumference, $\mathrm{AC}=$ abdominal

649 circumference, $\mathrm{CBC}=$ cannon bone circumference, $\mathrm{RW}=$ rump width, $\mathrm{CW}=$ chest

650 width, SE of estimates are in parentheses 


\section{Table 3. Significant SNPs and associated genes for seven body size traits}

\begin{tabular}{|c|c|c|c|c|c|c|c|}
\hline Trait $^{1}$ & $\begin{array}{l}\text { Chrom } \\
\text { osome }\end{array}$ & Position (bp) & P_value & $\begin{array}{l}\text { SNP effect } \\
(\%)\end{array}$ & Gene & Distance & Gene function \\
\hline \multirow[t]{9}{*}{$\mathrm{BL}$} & 6 & 5671575 & $2.35 \mathrm{E}-07$ & 0.00186 & CDH13 & +13217 & cadherin 13 \\
\hline & 1 & 6435744 & $1.5 \mathrm{E}-06$ & 0.00017 & NA & & NA \\
\hline & 1 & 6472959 & $1.5 \mathrm{E}-06$ & 0.00080 & PRKN & +38323 & parkin RBR E3 ubiquitin protein ligase \\
\hline & 17 & 33632497 & $2.62 \mathrm{E}-06$ & 0.00289 & $\begin{array}{c}\text { ENSSSCG00000028 } \\
461\end{array}$ & -47405 & signal regulatory protein alpha \\
\hline & 13 & 25520933 & 4.45E-06 & 0.00004 & ULK4 & -8396 & unc-51 like kinase 4 \\
\hline & 16 & 1276330 & 4.57E-06 & 0.00031 & NA & & NA \\
\hline & 14 & 137476010 & $6.47 \mathrm{E}-06$ & 0.00054 & NA & & NA \\
\hline & 8 & 28933773 & $7.46 \mathrm{E}-06$ & 0.00144 & NWD2 & -23316 & NACHT and WD repeat domain containing 2 \\
\hline & 13 & 166328893 & 8.39E-06 & 0.00039 & NA & & NA \\
\hline \multirow[t]{6}{*}{$\mathrm{BH}$} & 16 & 886074 & 2.84E-06 & 0.00817 & CTNND2 & +28239 & alpha-2-macroglobulin like 1 \\
\hline & 8 & 7942460 & $3.01 \mathrm{E}-06$ & 0.00083 & NA & & NA \\
\hline & 3 & 26586077 & 4.62E-06 & 0.00117 & CLEC19A & -45911 & C-type lectin domain containing 19A \\
\hline & 5 & 62690928 & $6.5 \mathrm{E}-06$ & 0.00004 & A2ML1 & -42827 & alpha-2-macroglobulin like 1 \\
\hline & 4 & 128701315 & $7.54 \mathrm{E}-06$ & 0.00152 & NA & & NA \\
\hline & 14 & 33580513 & $9.85 \mathrm{E}-06$ & 0.00060 & HSPB8 & +45615 & heat shock protein family B (small) member \\
\hline \multirow[t]{13}{*}{$\mathrm{CBC}$} & 4 & 117759672 & $2.16 \mathrm{E}-07$ & 0.00279 & CDC14A & -34935 & cell division cycle $14 \mathrm{~A}$ \\
\hline & 13 & 182971424 & $1.83 \mathrm{E}-06$ & 0.00420 & TMPRSS15 & -29625 & transmembrane serine protease 15 \\
\hline & 17 & 12868538 & $1.85 \mathrm{E}-06$ & 0.00635 & PSD3 & -43049 & pleckstrin and Sec7 domain containing 3 \\
\hline & 1 & 1201299 & 2.3E-06 & 0.00025 & ENSSSCG00000041 & -47914 & NA \\
\hline & 1 & 1205821 & 2.3E-06 & 0.00018 & \multicolumn{2}{|c|}{$\begin{array}{c}\text { ENSSSCG00000050 } \\
693\end{array}$} & NA \\
\hline & 1 & 1220233 & 2.3E-06 & 0.00039 & \multicolumn{2}{|c|}{$\begin{array}{c}\text { ENSSSCG00000045 } \\
916\end{array}$} & NA \\
\hline & 1 & 1367723 & 2.3E-06 & 0.00064 & \multicolumn{2}{|c|}{$\begin{array}{c}\text { ENSSSCG00000043 } \\
714\end{array}+5537$} & NA \\
\hline & 18 & 21663467 & 0.000003 & 0.00400 & GRM8 & -14659 & glutamate metabotropic receptor 8 \\
\hline & 14 & 9698552 & $3.19 \mathrm{E}-06$ & 0.00026 & \multicolumn{2}{|l|}{ ENSSSCG00000049 } & NA \\
\hline & 5 & 7020488 & $3.46 \mathrm{E}-06$ & 0.00023 & PMM1 & -49963 & phosphomannomutase 1 \\
\hline & 12 & 50490164 & 4.08E-06 & 0.00233 & SPNS3 & -47230 & sphingolipid transporter 3 (putative) \\
\hline & 3 & 12869355 & 4.1E-06 & 0.00259 & \multicolumn{2}{|l|}{$\begin{array}{c}\text { ENSSSCG00000036 } \\
217\end{array}$} & NA \\
\hline & 4 & 10221008 & $5.38 \mathrm{E}-06$ & 0.00076 & ASAP1 & -37722 & ArfGAP with $\mathrm{SH} 3$ domain, ankyrin repeat \\
\hline
\end{tabular}




$\begin{array}{ccccccc}124456560 & 5.76 \mathrm{E}-06 & 0.00036 & \text { PRR16 } & +6055 & \text { proline rich } 16 \\ 1 & 13806583 & 7.02 \mathrm{E}-06 & 0.00142 & \text { ENSSSCG00000004 } & \\ & & & 082527 & \text { NA }\end{array}$

\begin{tabular}{|c|c|c|c|c|c|c|c|}
\hline \multicolumn{8}{|c|}{652} \\
\hline Trait $^{1}$ & $\begin{array}{l}\text { Chrom } \\
\text { osome }\end{array}$ & Position (bp) & P_value & $\begin{array}{c}\text { SNP effect } \\
(\%)\end{array}$ & Gene & Distance & Gene function \\
\hline \multirow[t]{8}{*}{$\mathrm{AC}$} & 8 & 3249196 & $1.88 \mathrm{E}-06$ & 0.00134 & AFAP1 & -46660 & actin filament associated protein 1 \\
\hline & 9 & 14578071 & $2.31 \mathrm{E}-06$ & 0.00128 & NA & & NA \\
\hline & 14 & 13670622 & $2.71 \mathrm{E}-06$ & 0.00048 & PRSS55 & -4581 & serine protease 55 \\
\hline & 5 & 15137502 & $2.96 \mathrm{E}-06$ & 0.00513 & RHEBL1 & -39837 & RHEB like 1 \\
\hline & 4 & 5362087 & $4.48 \mathrm{E}-06$ & 0.00139 & $\begin{array}{c}\text { ENSSSCG0000004493 } \\
7\end{array}$ & +36176 & NA \\
\hline & 7 & 26363076 & $5.4 \mathrm{E}-06$ & 0.00093 & NA & & NA \\
\hline & 14 & 43227411 & $5.77 \mathrm{E}-06$ & 0.00024 & $\begin{array}{c}\text { ENSSSCG0000003338 } \\
5\end{array}$ & -49062 & KIAA1671 ortholog \\
\hline & 16 & 522752 & $6.96 \mathrm{E}-06$ & 0.00014 & CTNND2 & -3796 & catenin delta 2 \\
\hline \multirow[t]{16}{*}{$\mathrm{CC}$} & 3 & 63528527 & $1.32 \mathrm{E}-07$ & 0.00015 & $\begin{array}{c}\text { ENSSSCG0000000825 } \\
0\end{array}$ & -41861 & catenin alpha 2 \\
\hline & 6 & 19429624 & $3.27 \mathrm{E}-07$ & 0.00022 & Metazoa_SRP & -49801 & Metazoan signal recognition particle \\
\hline & 1 & 3149903 & $7.78 \mathrm{E}-07$ & 0.00047 & PDE10A & -28771 & phosphodiesterase $10 \mathrm{~A}$ \\
\hline & 6 & 120477523 & $1.95 \mathrm{E}-06$ & 0.00173 & FHOD3 & -40874 & formin homology 2 domain containi \\
\hline & 10 & 56219300 & $2.01 \mathrm{E}-06$ & 0.00203 & ITGB1 & -46293 & integrin subunit beta 1 \\
\hline & 17 & 18990746 & $2.63 \mathrm{E}-06$ & 0.00004 & ANKEF1 & -32351 & ankyrin repeat and EF-hand domain \\
\hline & 17 & 18997949 & $2.63 \mathrm{E}-06$ & 0.00008 & ANKEF1 & -37701 & ankyrin repeat and EF-hand domain \\
\hline & 2 & 122228151 & $3.03 \mathrm{E}-06$ & 0.00105 & $\begin{array}{c}\text { ENSSSCG0000005134 } \\
3\end{array}$ & -14167 & NA \\
\hline & 2 & 122235537 & 3.03E-06 & 0.00074 & $\begin{array}{c}\text { ENSSSCG0000005134 } \\
3\end{array}$ & -21553 & NA \\
\hline & 16 & 33630686 & $3.58 \mathrm{E}-06$ & 0.00018 & NA & & NA \\
\hline & 16 & 33638300 & $3.58 \mathrm{E}-06$ & 0.00079 & NA & & NA \\
\hline & 16 & 5533970 & 4.64E-06 & 0.00394 & $\begin{array}{c}\text { ENSSSCG0000001679 } \\
1\end{array}$ & +16579 & NA \\
\hline & 12 & 5297390 & $5.41 \mathrm{E}-06$ & 0.00092 & RNF157 & -48707 & ring finger protein 157 \\
\hline & 4 & 64552365 & 5.7E-06 & 0.00666 & $\begin{array}{c}\text { ENSSSCG0000004202 } \\
9\end{array}$ & -24706 & NA \\
\hline & 10 & 43341283 & $7.22 \mathrm{E}-06$ & 0.00064 & CUBN & -39687 & cubilin \\
\hline & 8 & 21799389 & 8.84E-06 & 0.00030 & $\begin{array}{c}\text { ENSSSCG0000005098 } \\
4\end{array}$ & -18261 & NA \\
\hline
\end{tabular}




\begin{tabular}{|c|c|c|c|c|c|c|c|}
\hline & 10 & 60737384 & $9.42 \mathrm{E}-06$ & 0.00449 & $\begin{array}{c}\text { ENSSSCG0000001112 } \\
1\end{array}$ & -24200 & CUGBP Elav-like family member \\
\hline \multirow[t]{9}{*}{ RW } & 8 & 137165913 & $5.64 \mathrm{E}-07$ & 0.00049 & NA & & NA \\
\hline & 9 & 2330339 & $2.74 \mathrm{E}-06$ & 0.00381 & SYT9 & -11533 & synaptotagmin 9 \\
\hline & 1 & 38033383 & 4.19E-06 & 0.00149 & NKAIN2 & -12912 & sodium/potassium transporting ATI \\
\hline & 3 & 63682227 & $5.03 \mathrm{E}-06$ & 0.00010 & NA & & NA \\
\hline & 11 & 32555905 & $6.78 \mathrm{E}-06$ & 0.00015 & DIAPH3 & +46764 & diaphanous related formin 3 \\
\hline & 16 & 48600234 & 7.1E-06 & 0.00118 & $\begin{array}{c}\text { ENSSSCG0000004608 } \\
5\end{array}$ & -23005 & NA \\
\hline & 16 & 48696355 & 7.1E-06 & 0.00155 & $\begin{array}{c}\text { ENSSSCG0000003988 } \\
3\end{array}$ & +49947 & NA \\
\hline & 1 & 100210738 & 7.97E-06 & 0.00095 & MAPK4 & +8278 & mitogen-activated protein kinase 4 \\
\hline & 1 & 100335688 & 7.97E-06 & 0.00017 & MAPK4 & -49706 & mitogen-activated protein kinase 4 \\
\hline \multirow[t]{18}{*}{$\mathrm{CW}$} & 8 & 132277288 & 8.17E-07 & 0.00009 & PTPN13 & -27877 & protein tyrosine phosphatase non-re \\
\hline & & & & & MAPK10 & -27281 & mitogen-activated protein kinase 10 \\
\hline & 7 & 115471416 & $9.52 \mathrm{E}-07$ & 0.00653 & PPP4R4 & -18233 & protein phosphatase 4 regulatory su \\
\hline & 14 & 37118119 & $1.09 \mathrm{E}-06$ & 0.00289 & $\begin{array}{c}\text { ENSSSCG0000005178 } \\
6\end{array}$ & -2275 & NA \\
\hline & 14 & 37165658 & $1.09 \mathrm{E}-06$ & 0.00051 & $\begin{array}{c}\text { ENSSSCG0000005178 } \\
6\end{array}$ & -49874 & NA \\
\hline & 14 & 37230969 & $1.09 \mathrm{E}-06$ & 0.00039 & $\begin{array}{c}\text { ENSSSCG0000005178 } \\
6\end{array}$ & -9755 & NA \\
\hline & 2 & 80016213 & $2.07 \mathrm{E}-06$ & 0.00192 & COL23A1 & -46865 & collagen type XXIII alpha 1 chain \\
\hline & 14 & 139878474 & $2.34 \mathrm{E}-06$ & 0.00437 & TCERG1L & -46513 & transcription elongation regulator 1 \\
\hline & 12 & 49725382 & $2.67 \mathrm{E}-06$ & 0.00112 & TRPV1 & -33424 & transient receptor potential cation $\mathrm{cl}$ \\
\hline & 16 & 35012960 & $3.46 \mathrm{E}-06$ & 0.00029 & DDX4 & -46102 & DEAD-box helicase 4 \\
\hline & 7 & 115132809 & 4.65E-06 & 0.00069 & $\begin{array}{c}\text { ENSSSCG0000000246 } \\
4\end{array}$ & -31787 & proline rich membrane anchor 1 \\
\hline & 16 & 73800572 & $4.82 \mathrm{E}-06$ & 0.00153 & U6 & +41611 & U6 spliceosomal RNA \\
\hline & 16 & 73812833 & 4.82E-06 & 0.00172 & U6 & +29350 & U6 spliceosomal RNA \\
\hline & 16 & 73816240 & 4.82E-06 & 0.00175 & U6 & +25343 & U6 spliceosomal RNA \\
\hline & 9 & 107845695 & $5.74 \mathrm{E}-06$ & 0.00014 & $\begin{array}{c}\text { ENSSSCG0000003290 } \\
5\end{array}$ & -7136 & NA \\
\hline & 8 & 76456715 & $6.42 \mathrm{E}-06$ & 0.00057 & $\begin{array}{c}\text { ENSSSCG0000004227 } \\
3\end{array}$ & -45304 & NA \\
\hline & 3 & 131731345 & $6.59 \mathrm{E}-06$ & 0.00001 & $\begin{array}{c}\text { ENSSSCG0000004975 } \\
1\end{array}$ & -23407 & NA \\
\hline & 3 & 131738702 & $6.59 \mathrm{E}-06$ & 0.00016 & ENSSSCG0000004975 & -30764 & NA \\
\hline
\end{tabular}


1

\begin{tabular}{|c|c|c|c|c|c|c|}
\hline 3 & 131744661 & $6.59 \mathrm{E}-06$ & 0.00016 & $\begin{array}{c}\text { ENSSSCG0000004975 } \\
1\end{array}$ & -36723 & NA \\
\hline 3 & 131756951 & $6.59 \mathrm{E}-06$ & 0.00025 & $\begin{array}{c}\text { ENSSSCG0000004975 } \\
1\end{array}$ & -43896 & NA \\
\hline 3 & 131758601 & $6.59 \mathrm{E}-06$ & 0.00024 & $\begin{array}{c}\text { ENSSSCG0000004975 } \\
1\end{array}$ & -45546 & NA \\
\hline 5 & 61521505 & $7.76 \mathrm{E}-06$ & 0.00033 & $\begin{array}{c}\text { ENSSSCG0000003340 } \\
3\end{array}$ & -14845 & C-type lectin domain family 7 mem \\
\hline 16 & 67601687 & $8.2 \mathrm{E}-06$ & 0.00052 & $\begin{array}{c}\text { ENSSSCG0000004922 } \\
9\end{array}$ & -42425 & NA \\
\hline 7 & 92897102 & 4.65E-06 & 0.00109 & HMGA1 & +25885 & high mobility group AT-hook 1 \\
\hline 15 & 11796106 & $9.96 \mathrm{E}-06$ & 0.00074 & NA & & NA \\
\hline
\end{tabular}

653 Note: ${ }^{1} \mathrm{BL}=$ body length, $\mathrm{BH}=$ body height, $\mathrm{CC}=$ chest circumference, $\mathrm{AC}=$ abdominal

654 circumference, $\mathrm{CBC}=$ cannon bone circumference, $\mathrm{RW}=$ rump width, $\mathrm{CW}=$ chest

655 width, gene effect= proportion of genetic variance explained

656 Table 4 Overview of ssGWAS location for the percentage that explains the

657 proportion of genetic variance

\begin{tabular}{|c|c|c|c|c|c|}
\hline Trait $^{1}$ & $\begin{array}{c}20 \text { SNPs distributions of } \\
\text { maximum effect }\end{array}$ & $\begin{array}{c}\text { SNPs of the maximum } \\
\text { effect }\end{array}$ & $\begin{array}{c}\text { Top } 20 \\
\text { SNPs } \\
\text { effect }(\%)\end{array}$ & $\begin{array}{c}\text { Number } \\
\text { of } \\
\text { nearest } \\
\text { gene }\end{array}$ & $\begin{array}{c}\text { Candidate } \\
\text { gene }\end{array}$ \\
\hline $\mathrm{BL}$ & SSC17 & 17_7477978 & 0.117 & 4 & \\
\hline $\mathrm{BH}$ & SSC2、SSC5、SSC16 & 2_46827557 & 0.08 .7 & 8 & SIL1 \\
\hline $\mathrm{CBC}$ & SSC7、SSC4 & 7_55099416 & 0.101 & 17 & TRAPPC9 \\
\hline $\mathrm{AC}$ & $\mathrm{SSC} 12$ & 12_53181656 & 0.128 & 8 & $\begin{array}{c}\text { KDM6B } \\
\text { CHD3 }\end{array}$ \\
\hline $\mathrm{CC}$ & $\mathrm{SSC} 12$ & 12_53169477 & 0.129 & 8 & $\begin{array}{c}\text { KDM6B } \\
\text { CHD3 }\end{array}$ \\
\hline RW & $\begin{array}{c}\text { SSC6、SSC7、SSC12 } \\
\text { SSC13、SSC17 }\end{array}$ & 17_13172524 & 0.099 & 15 & MUC13 \\
\hline $\mathrm{CW}$ & $\begin{array}{c}\text { SSC6、SSC7、SSC13、 } \\
\text { SSC17 }\end{array}$ & 6_39554872 & 0.070 & 28 & MUC13 \\
\hline
\end{tabular}

658 Note: ${ }^{1} \mathrm{BL}=$ body length, $\mathrm{BH}=$ body height, $\mathrm{CC}=$ chest circumference, $\mathrm{AC}=$ abdominal

659 circumference, $\mathrm{CBC}=$ cannon bone circumference, $\mathrm{RW}=$ rump width, $\mathrm{CW}=$ chest

660 width, gene effect= proportion of genetic variance explained

661

662 Additional files 


\section{Additional file Table S1}

664 Format: DOCX

665 Title: Each trait explains the 20 SNPs with the greatest genetic variance. 


\section{Figures}
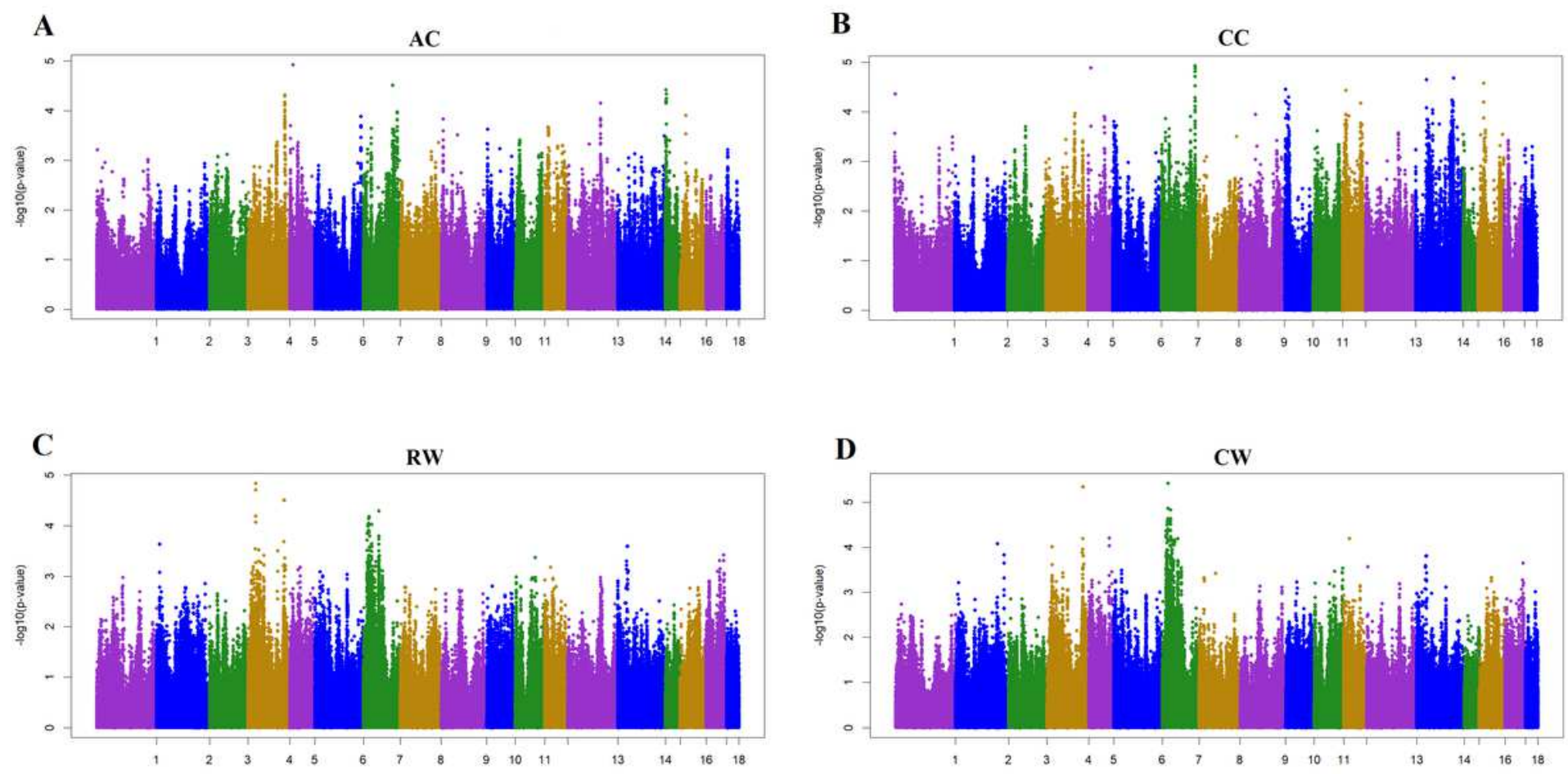

\section{Figure 1}

Manhattan plot of the genome-wide association study on three body size traits by using single-trait model ssGWAS. BL, Body length; BH, Body height; $\mathrm{CBC}$, Cannon bone circumference. In the Manhattan plots, negative log10 P-values of the quantified SNPs were plotted against their genomic positions. The $x-$ axis represents the chromosomes, and the $y$-axis represents the observed-log10(P-value). Different colors indicate various chromosomes. Each trait has a significant threshold of FDR adjusted, for (A) BL, it was $9.26 \times 10-6$. Similarly, (B) BH was $1.08 \times 10-5$, and (D) CBC was $1.02 \times 10-5$. 
A

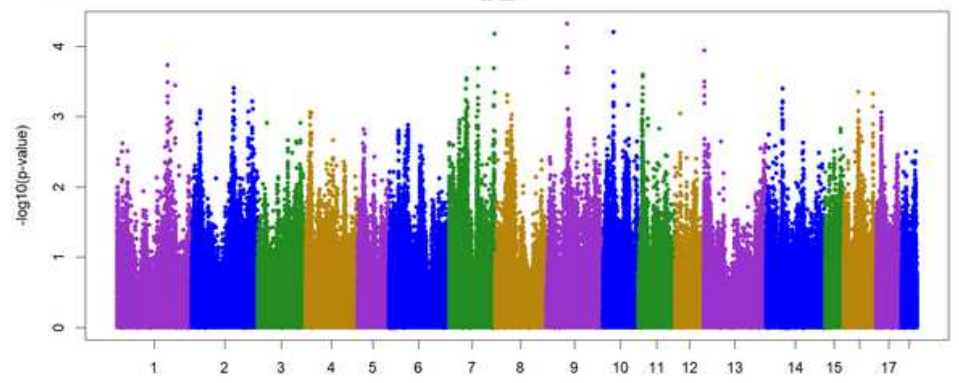

B

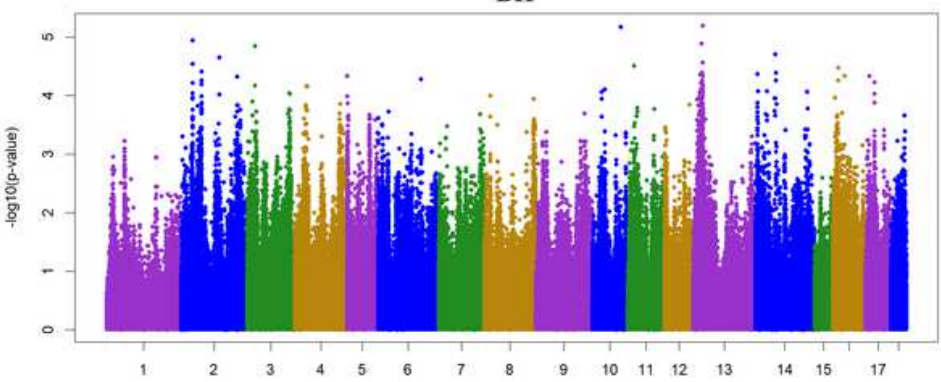

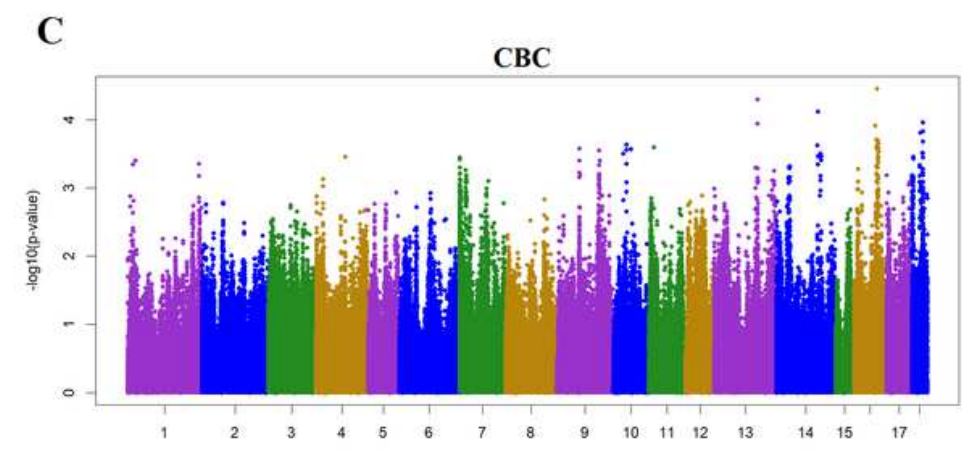

Figure 2

Manhattan plot of the genome-wide association study on four body size traits by using two-trait model ssGWAS. AC, Abdominal circumference; CC, Chest circumference; RW, Rump width; CW, Chest width. AC and CC are a pair of traits, RW and CW are a pair of traits. In the Manhattan plots, negative log10 P-values of the quantified SNPs were plotted against their genomic positions. The $x$-axis represents the chromosomes, and the $y$-axis represents the observed -log10(P-value). Different colors indicate various chromosomes. Each trait has a significant threshold of FDR adjusted, for (A) AC, it was $9.74 \times 10-6$. Similarly, (B) CC was $1.05 \times 10-5$, (C) RW was $9.60 \times 10-6$, and (D) CW was $1.01 \times 10-5$. 
A
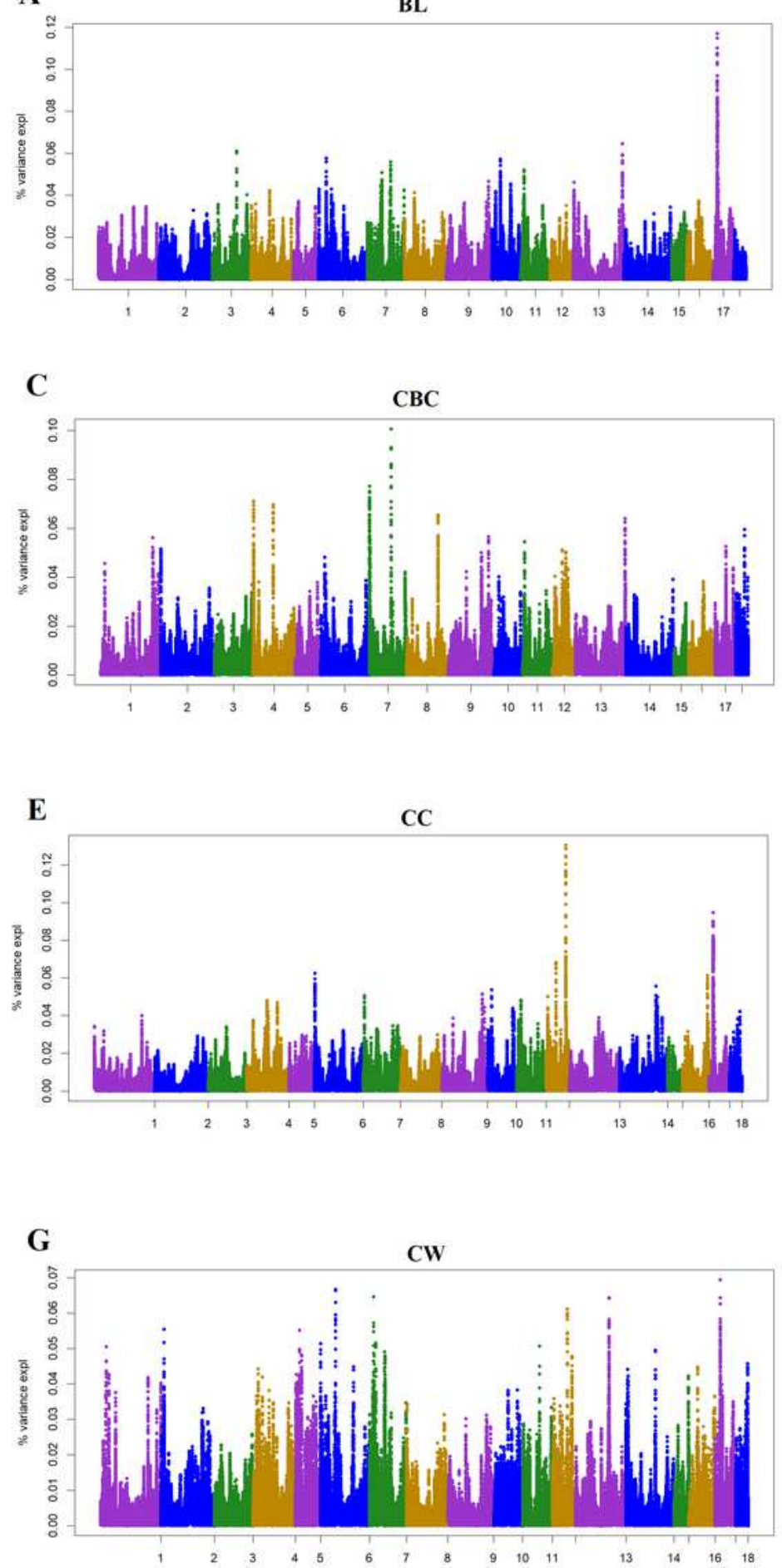
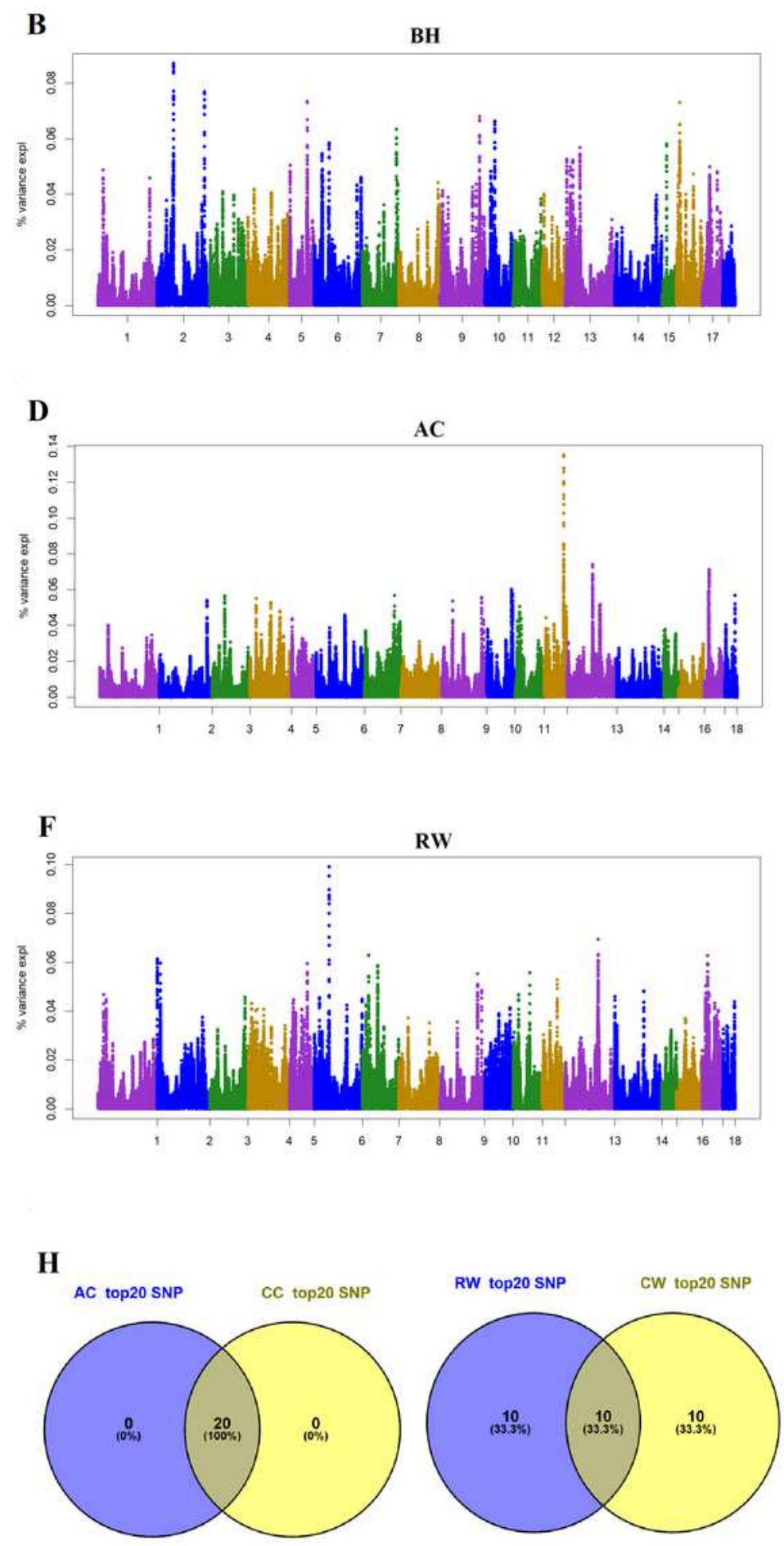

Figure 3

Manhattan plot of the genome-wide association study on seven body size traits and Venn plot of SNPs according to the contribution of SNP to genetic variance by using SsGWAS. BL, Body length; $B H, B o d y$ height; $C B C$, Cannon bone circumference; $A C$, Abdominal circumference; $C C$, Chest circumference; RW, Rump width; $\mathrm{CW}$, Chest width. $\mathrm{BL}, \mathrm{BH}$ and $\mathrm{CBC}$ were single-trait models $\triangle \mathrm{AC}, \mathrm{CC}, \mathrm{RW}$ and $\mathrm{CW}$ were two-trait models. AC and $C C$ are a pair of traits, RW and $C W$ are a pair of traits. In the Manhattan plots $(A-G)$, the 
proportion of genetic variance of the quantified SNPs were plotted against their genomic positions. The xaxis represents the chromosomes, and the $y$-axis represents the percentage of SNP explaining the genetic variance. Different colors indicate different chromosomes. Venn plot $(\mathrm{H})$ of SNPs for the two pairs of body size traits, AC and CC, RW and CW are a pair of traits, respectively.

\section{Supplementary Files}

This is a list of supplementary files associated with this preprint. Click to download.

- SupplementaryTable.docx 